\title{
A Methodology for Developing Agent Based Systems for Enterprise Integration
}

\author{
E. A. Kendall, M. T. Malkoun and C. H. Jiang \\ Department of Computer Systems Engineering \\ Royal Melbourne Institute of Technology \\ GPO Box 2476V, Melbourne, VIC 3001 AUSTRALIA \\ email address: kendall@rmit.edu.au
}

\begin{abstract}
One of the most significant opportunities for enterprise integration is the recent advancement of agent based systems. However, before agents can be widely used, a methodology must be established for the development of these systems. This paper outlines a methodology for the software engineering of agent based systems. The methodology is based upon the IDEF (ICAM Definition) approach for workflow modelling, the CIMOSA enterprise modelling framework, and the use case driven approach to object oriented software engineering. The methodology is illustrated by a case study in the area of discrete parts manufacturing.
\end{abstract}

Keywords

Agent based systems, object oriented software engineering, ICAM.

\subsection{INTRODUCTION}

Agent based systems have been applied to enterprise integration (Pan and Tenenbaum, 1991), concurrent engineering (Tenenbaum et al., 1992), and manufacturing (Kwok and Norrie, 1993). These systems exhibit significant advances in distributed problem solving.

Before agents can be widely used for enterprise engineering, a methodology must be established for their development. The need for software engineering methodologies for agent based systems has been described elsewhere. Work by (Huntbach et al., 1995) indicated that an agent is a process or a data store in a data flow diagram (Cohen and Ringwood, 1995). Agent behavior is actually quite different from a process or a data store. The need to consider work flow and not just data flow for agents has been discussed (Klein, M., 1995), as agents cooperate much as individuals do in organizations. (Klein, M., 1995) provides the following table for a comparison of dataflow and workflow representations and details that workflow representations are more suitable.

\begin{tabular}{|l|l|}
\hline Dataflow & Workflow \\
\hline Between computer systems & Between human participants \\
\hline Pass information & Pass tasks \\
\hline Pre-defined and rigid & Can have exceptions \\
\hline First in First Out queues & Reasoning about ordering/merging \\
\hline Systems are interchangeable & People have unique skills and positions \\
\hline Requires input data & Requires task execution environment \\
\hline
\end{tabular}


In this paper, existing methodologies for workflow and object oriented analysis and design are extended to encompass agent oriented systems. The research proposes that as agents must be implemented in software, a workflow representation alone can not be used. An object oriented software engineering methodology is employed, but the object oriented model has an equivalent workflow representation that is discussed. In particular, the IDEF methodology, the CIMOSA modelling framework, and the use case object oriented approach are utilized. These tools have been employed because they capture the work flow and active nature of an agent system. They are also mainstream approaches that are widely used.

\subsection{BACKGROUND}

\subsection{Agents}

There are two views of agents: weak and strong (Wooldridge and Jennings, 1995). A weak definition of agency is items i) through iv) while a strong definition adds one of v) through vii):

i) autonomous - agents operate without direct intervention ii) social - agents interact with other agents iii) reactive - agents perceive their environment and respond to changes that occur iv) pro-active - agents affect their environment v) mentalistic notions - an agent has knowledge, and desires, intentions, and commitments vi) rationality - an agent performs actions which further its goals vii) veracity and viii) adaptability or learning.

Strong agents are also termed reflective in that they reflect on their behavior rather than merely reacting to stimuli or changes. Strong agents are emphasized in this research, although only items i) through vi) above are considered. Strong agents reason about their beliefs to select a plan that could achieve their stated goals. Beliefs in reflective agent oriented systems are primarily represented in extensions to first order predicate calculus. Two examples are dMARS (Distributed Multiagent Reasoning System) (Brenton, 1995) and KIF (Knowledge Interchange Format) (Genesereth, Fikes, et. al, 1992).

Agent pro-active behavior is represented in a plan library. An agent selects a plan from the plan library on the basis of the goals that are to be accomplished. A plan is instantiated when a triggering event occurs that satisfies its invocation and context conditions. An instantiated plan is an intention. The body of a plan is tasks that can be subgoals, actions, assertions to and retractions from the belief database, and queries and messages to other agents. When an intention is formed, these tasks are executed by the agent in an effort to reach the stated goal.

Agents operate in a distributed environment; therefore, agents require mechanisms that allow them to communicate with other agents. KQML (Finin, Weber, et al, 1993) is a language for communication among agents. With KQML, agents can query for and offer information, along with other options. Agents must cooperate and negotiate with each other. In negotiation, agents engage in conversations that can be represented in speech acts and state machines. Languages have been developed to support this. COOL is an extension to KQML (Barbuceanu and Fox, 1995), and with it agents can make proposals and counterproposals, accept and reject goals, and notify other agents of goal cancellation, satisfaction, or failure. Another language, AgenTalk (Kuwabara, Ishida, and Osato, 1995), has an inheritance or specialization mechanism for introducing new protocols as extensions of predefined ones. Work by (Dunskus et al., 1995) has resulted in a preliminary categorization of agents. Selectors, evaluators, and other types have been described as single function agents. This 
approach is valuable in that it addresses given applications at a more abstract level. Basic types of negotiation between these agents, in particular conflict resolution (Klein, 1991), have also been identified.

\subsection{Object Oriented Software Engineering}

The notion of an object encompasses all of the following features (Booch, 1994):

i) Compound - an object can have properties that are objects themselves ii) Inheritance - an object type can have subtypes iii) Encapsulation - an object can hide data and behavior iv) Identity - a system can distinguish between two objects v) State - an object's behavior is dependent on its history and vi) Behavior - an object can act and react

During object oriented analysis, an object oriented model is developed for a given application. The Object Modelling Technique (OMT) (Rumbaugh et al., 1991), consists of three models: object, dynamic and functional. The object model indicates objects and relationships. The dynamic model includes scenarios and event traces of object interactions. The functional model resembles a data flow diagram, with passive objects as data stores. State diagrams are used to provide an overall view and to gain insight into inheritance. Subclasses inherit the state diagram of a superclass, adding states and transitions. Therefore, the protocols of AgenTalk are objects that can be specialized using inheritance, as stated in (Kuwabara, Ishida, and Osato, 1995).

In the use case approach (Jacobson, 1992), a use case is a scenario of how users interact with the system. A user in a particular role is known as an actor, and each actor uses one or more use cases. Objects relevant to a use case are identified, and their dynamic behavior is analysed. One use case can extend another one, meaning that it is inserted into the first use case, adding to its definition. Extend relationships can be conditional, occurring under only certain circumstances. Use cases can also be described at varying levels of abstraction, and inheritance can be utilized for specialization. A use case may be abstract or concrete.

\subsection{Enterprise Modelling Methodologies}

The IDEF (ICAM Definition) method (Bravoco and Yadav, 1985a, 1985b) is a standard for requirements definition in manufacturing and other organizations. IDEF comprises three models: the function $\left(\mathrm{IDEF}_{0}\right)$, information $\left(\mathrm{IDEF}_{1}\right)$, and dynamic models $\left(\mathrm{IDEF}_{2}\right)$. In the function model, the organization is described in terms of a hierarchy of decisions, activities, or actions. Each function has a ICOM (Input, Control, Output, and Mechanism) representation.

The CIMOSA modelling framework (Kosanke, 1992) centers on types of major constructs. In CIMOSA, an enterprise activity is analogous to an IDEF function, except it can have control input and output, and resource or mechanism input and output. Both the CIMOSA and $\mathrm{IDEF}_{0}$ models can be taken to a high degree of detail, as shown in Figure 1. Here, an $\mathrm{IDEF}_{0}$ function includes decisions that are made on the basis of control input. Decide Action chooses a method or activity (Method 1 or Method 2), based upon the input and the control input. 


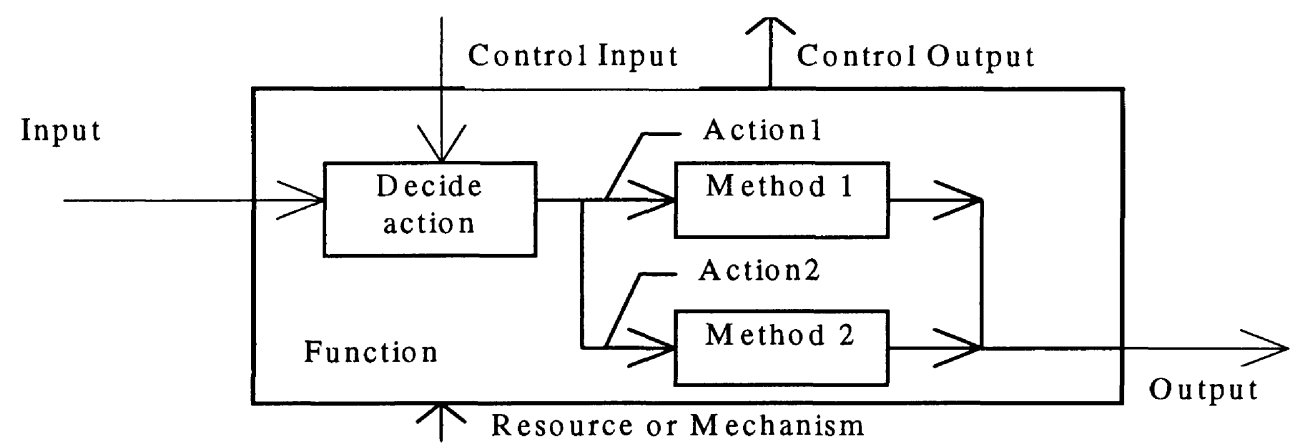

Figure 1 Detailed Function in $\mathrm{IDEF}_{0}$.

\subsection{APPROACH}

\subsection{Software Engineering and Enterprise Modelling Methodologies}

The three views of the IDEF methodology have counterparts in object oriented software engineering. If the $\mathrm{IDEF}_{0}$ function model is reexpressed in terms of an OMT functional model, active objects and passive objects (data stores) can be distinguished. With this approach, active input and output objects are identified, and mechanisms can also be modelled as active objects.

When use case and $\mathrm{IDEF}_{0}$ diagrams are completed to the same level, IDEF functions appear directly as a use case. In an $\mathrm{IDEF}_{0}$ diagram, use case actors would appear as resources or mechanisms. The objects that appear within the use case are the same as those found in the $\mathrm{IDEF}_{1}$ information model. Use case extensions are the same as multi-level (parent and child) $\mathrm{IDEF}_{0}$ diagrams. When actors and objects manage a given use case, they generate control information and pass it on as input to other components of the system. With this, policy or decision making actors and control objects carry out enterprise activities with control output.

\subsection{Agents and Objects}

Agents are real time, autonomous objects that carry out pro-active behavior. Each object sequentially executes methods, whereas an agent has automated reasoning that allows it to choose one alternative. An object responds to messages and events and executes its encoded behavior, step by step. Because agents are concurrent, the use of inheritance in agent software requires avoidance of the inheritance anomaly (Matsuoka and Yonezawa, 1993).

Synchronization behavior for a superclass will not necessarily provide accurate synchronization in a subclass; superclass synchronization behavior must often be redefined at the subclass level.

The distinctions between agents and objects can be summarized by the following:

Agents and objects: Compound, Encapsulation, Identity, State, Behavior.

Objects: Inheritance, Unstructured messages.

Agents: Reasoning, Pro-active behavior, Concurrency, Structured messages.

The first relationship between agents and objects is beliefs. Passive objects that store attributes frequently appear in belief databases (Georgeff and Ingrand, 1990). The Ontolingua project (Gruber et al., 1992) has produced software to translate frame based knowledge into a KIF beliefs database, and vice versa. (A frame, as an object, exhibits static aspects but differs 
in other ways.) Ontolingua can directly translate the static properties of objects that appear in an agent's belief database into KIF and other predicate calculus representations.

Objects with encapsulation and dynamic behavior can be utilized by agents to initiate and to carry out pro-active behavior. In this regard, objects can be sensors and effectors for agents. Objects in the environment impact the sensors and are impacted by the effectors, and all interaction is through messaging. Sensors message an agent with information about the environment that may impact the agent's beliefs and knowledge. These changes to the agent's beliefs may cause triggering events, instantiating a plan to an intention. Effectors carry out an agent's intentions, impacting objects in the environment.

Use cases depict how humans interact with a system, with each actor as a user in a certain role. Agents enter applications to augment or automate human reasoning. For a full requirements analysis, use cases must be specified for each scenario of the application. At this level of detail each use case maps to an actor's (or agent's) plan. Conditional extends relationships --- when one use case is inserted into another if certain circumstances exist --correspond to context conditions that determine when an agent's plan is to be intended.

When multiple actors appear in a use case, they collaborate and negotiate. In IDEF, resources collaborate by appearing in the same function or by exchanging input /output. In the agent system, the actors and resources become the agents and their negotiation becomes a coordination protocol. Use cases and protocols can be abstracted and specialized.

\subsection{An Agent Oriented Methodology}

Results are summarized in Figure 2 where Figure 1 is modified to indicate the corresponding agent. The inputs and outputs are the same, but the interior now shows an agent. The agent has beliefs, goals, plans, and an interpreter, with passive objects as beliefs. Instantiated plans become intentions via the interpreter. Three kinds of intentions are indicated. Task 1 involves coordination, task 3 invokes an effector, and task 2 impacts the beliefs. A use case extension diagram is depicted, and this translates into the agent's plans.

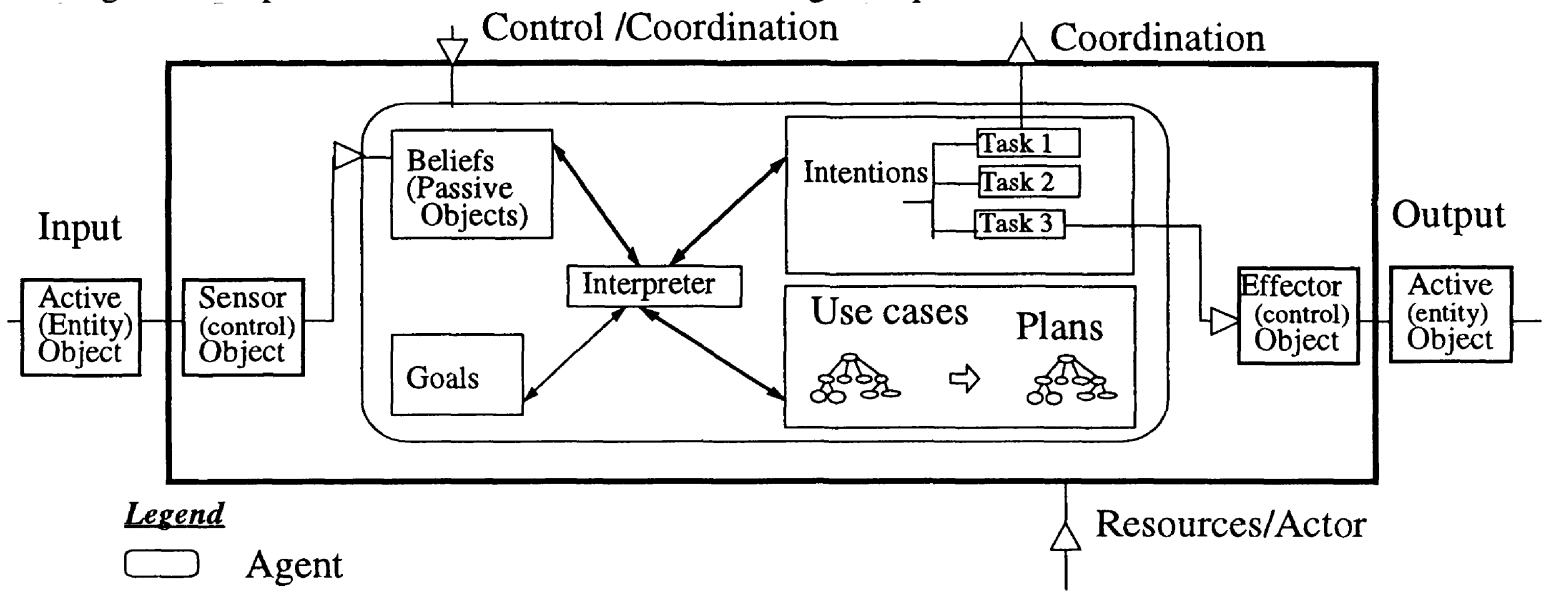

Figure 2 The Correspondence between Enterprise Activities, Use Cases and Agents. 
The correspondence between IDEF, use case and agent system components is then summarized in the following table.

\begin{tabular}{|c|c|c|}
\hline IDEF Model & Use Case & Agent Oriented System \\
\hline $\begin{array}{l}\text { Function w/ Control } \\
\text { Output }\end{array}$ & Use Case / Use Case Extension & Goal and Plan \\
\hline Functional Input & $\begin{array}{l}\text { - Entity Object } \\
\text { - Actor Input }\end{array}$ & $\begin{array}{l}\text { - Object via Sensor } \\
\text { - Belief }\end{array}$ \\
\hline Control Input & $\begin{array}{l}\text { - Object via Control Object } \\
\text { - Actor Input } \\
\text { - Use Case Extension }\end{array}$ & $\begin{array}{l}\text { - Object via Sensor } \\
\text { - Belief } \\
\text { - Context Condition }\end{array}$ \\
\hline Control Output & $\begin{array}{l}\text { - Object to Entity Object } \\
\text { - Control Object to Actor }\end{array}$ & $\begin{array}{l}\text { - Goal or Subgoal } \\
\text { - Effector to Object }\end{array}$ \\
\hline Functional Output & $\begin{array}{l}\text { - Object to Entity Object } \\
\text { - Control Object to Actor }\end{array}$ & - Effector to Coexisting Object \\
\hline Resource & Actor & Agent \\
\hline $\begin{array}{l}\text { Multiple resources } \\
\text { per function or } \\
\text { information } \\
\text { exchange }\end{array}$ & $\begin{array}{l}\text { - Multiple actors per use case } \\
\text { - Use case event trace } \\
\text { - Use case abstraction/ } \\
\text { specialization (inheritance) }\end{array}$ & $\begin{array}{l}\text { - Agent collaboration } \\
\text { - Coordination protocol } \\
\text { - Abstraction/ specialization } \\
\text { (inheritance) }\end{array}$ \\
\hline Information Model & Objects & - Beliefs and Objects \\
\hline
\end{tabular}

\subsection{CASE STUDY}

The case study is from discrete parts manufacturing. Customers place part orders, and the parts are selected for processing and grouped into batches. A batch is assigned to a machine for processing, and the cost of a batch is determined by the material used and wasted, and on handling and processing. There are high quality and normal quality batches. Normal parts can be formed on a high quality machine, but not vice versa.

\subsection{IDEF Model}

IDEF $_{0}$ diagrams are provided in Figures 3 and 4 . In Figure 3, forming is not shown, and the system has five functions. Input and output are indicated, along with resources and control. Control feedback is shown, as costing impacts the policies used for selection, batching, scheduling machines, and costing itself. These feedback paths are two way, with selection, batching and scheduling policies also impacting the cost evaluation policy.

Determination of the part selection policy is addressed in a level 2 IDEF $_{0}$ diagram. Part selection policy is determined on the basis of parts and material. Information supplied from the control output of costing is used, along with strategic knowledge. The selection policy is used to control part and sheet selection; the selection policy decision may also impact costing via control input. In CIMOSA, the selection policy functional output would be portrayed as a control output and the set selection policy function would be an enterprise activity. 


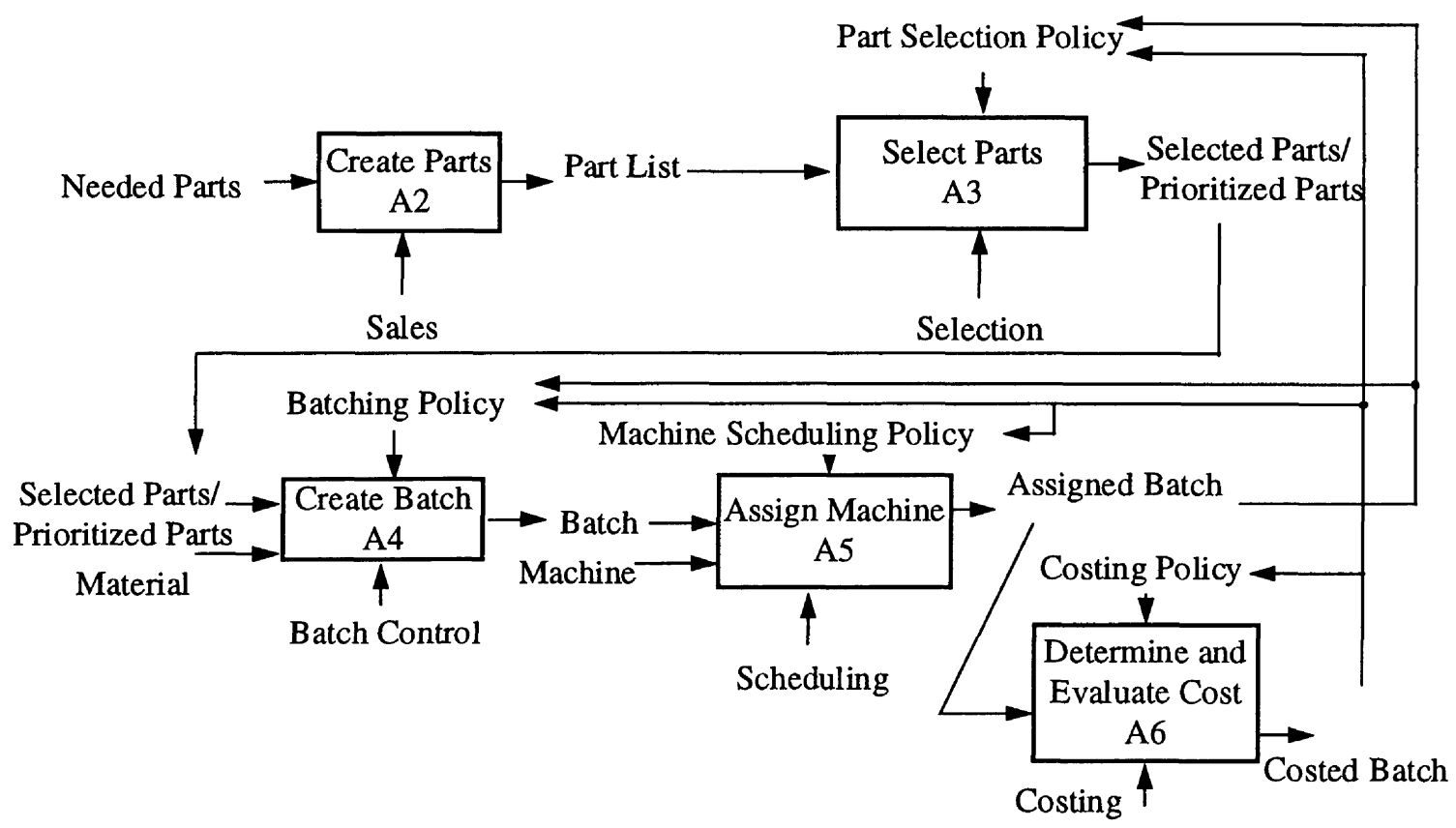

Figure 3 Level $1 \mathrm{IDEF}_{0}$ Function Model Overview of Case Study.

Selection policy determination monitors part due dates, qualities, and sizes, and input from costing. Simple selection policies select the first or the largest part with the earliest due date. Alternatively, parts may be selected because of the available material, and special orders may occur. Lastly, the selection policy may be based upon collaboration with costing, giving precedence to parts and sheets that are cost effective. Costing might recommend a sheet that is available but also large enough for cost effective handling, or a part that meets cost trends. If selection is able to satisfy these requests, this collaboration with costing involves rejecting past selections. If selection is unable to carry out these recommendations, this result must be fed back to costing. These possibilities are depicted in Figure 4, a level 3 IDEF $_{0}$ model. Level 3 diagrams for the Set Batching Policy, Set Scheduling Policy, and Evaluate Cost functions have also been produced but are omitted from this discussion for brevity.

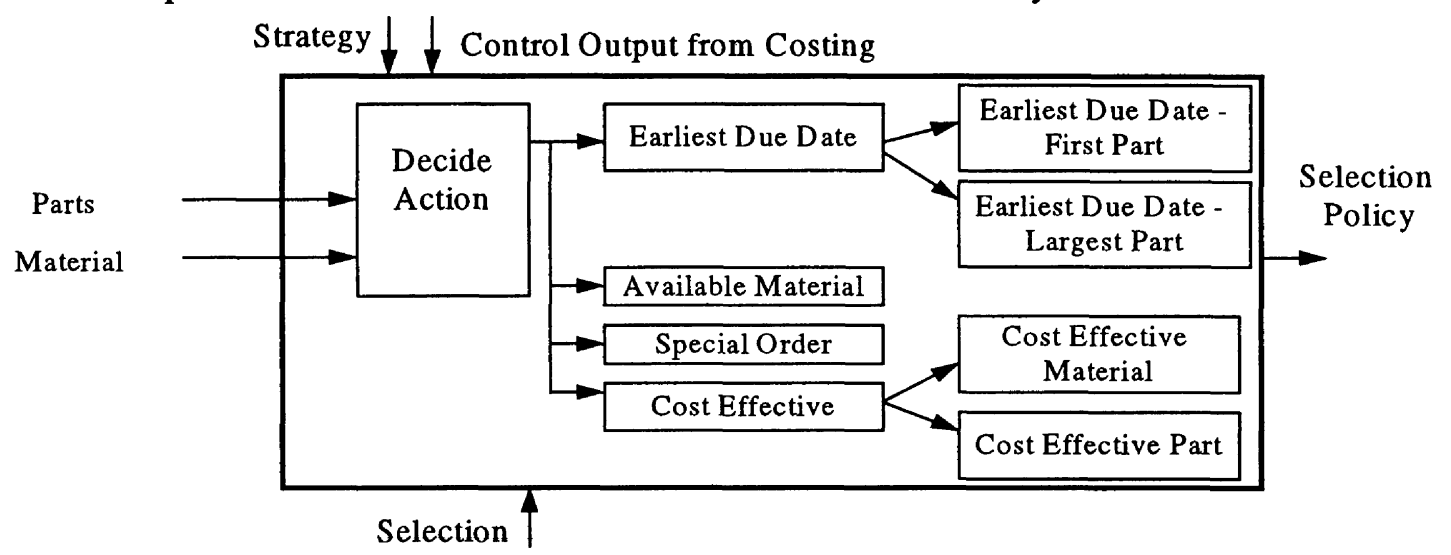

Figure 4: $\mathrm{IDEF}_{0}$ Level 3 Diagram of Set Selection Policy. 


\subsection{Use Case Model}

Use case representations are in Figure 5. The stick figures are actors while each ellipse is a use case. Uses relationships are shown by dashed arrows; an actor uses a case if it appears in the scenarios. Extends relationships are indicated by a solid arrow, and one use case extends another if it adds scenarios under certain condiations. If an actor uses a base use case it also uses the extensions. Figure 5 depicts the nine IDEF functions and the five resources. The four level 2 IDEF use cases extend the five level 1 use cases. Selection sets selection policy and selects parts, Batching sets batching policy and creates the batches. Assigning machines and setting scheduling policy are used by Scheduling. As the Costing actor gives input for policy selection, it uses these cases. Likewise, four actors collaborate for the cost evaluation.

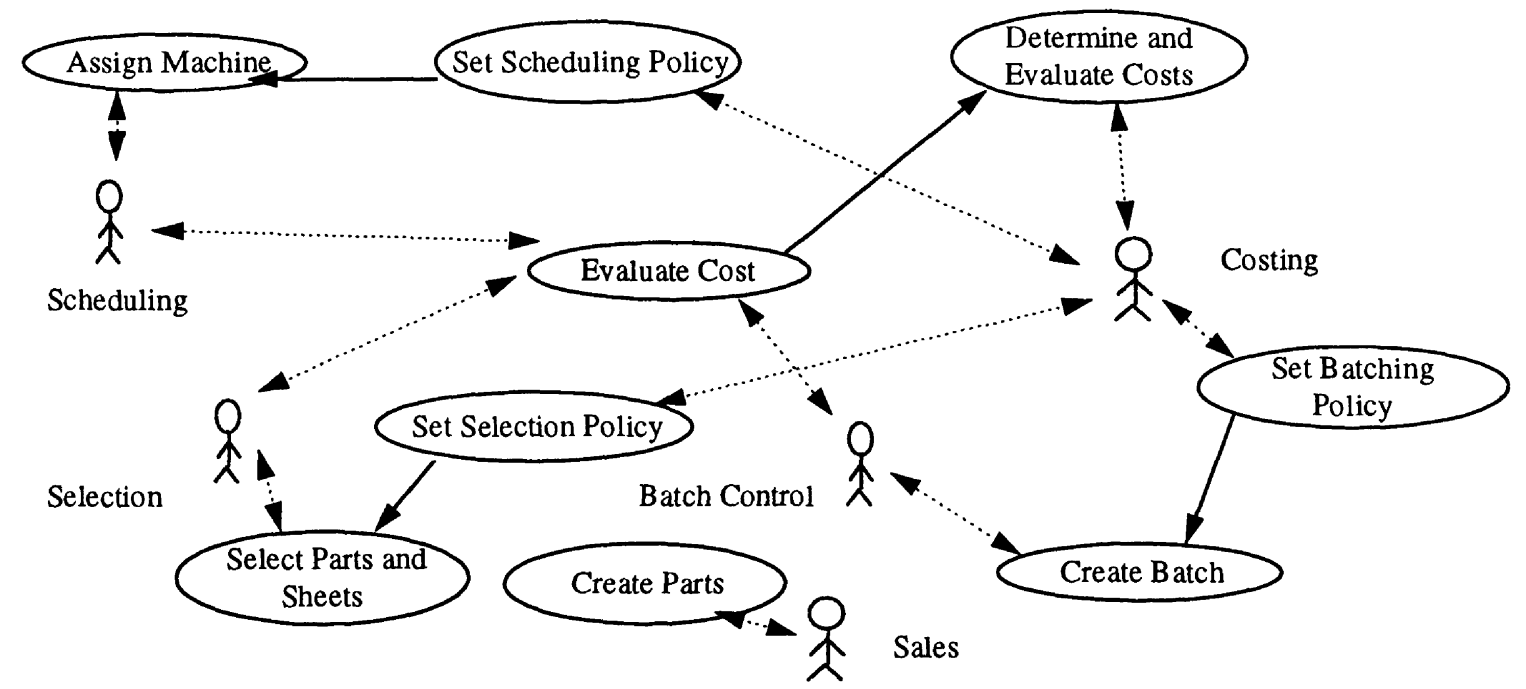

Figure 5 Use Cases for the Application.

Figure 6 shows use cases extending Set Selection Policy as the functions in the level 3 IDEF diagram are extensions of higher level functions. The Set Part Selection Policy use case is extended by Earliest Due Date, Special Order, Available Material, and Cost Effective. Earliest Due Date is further, while Cost Effective is extended to Cost Effective Part and Material. The level 3 use case extensions are conditional. Use case extensions for batching, scheduling, and costing policy determinations are similar.

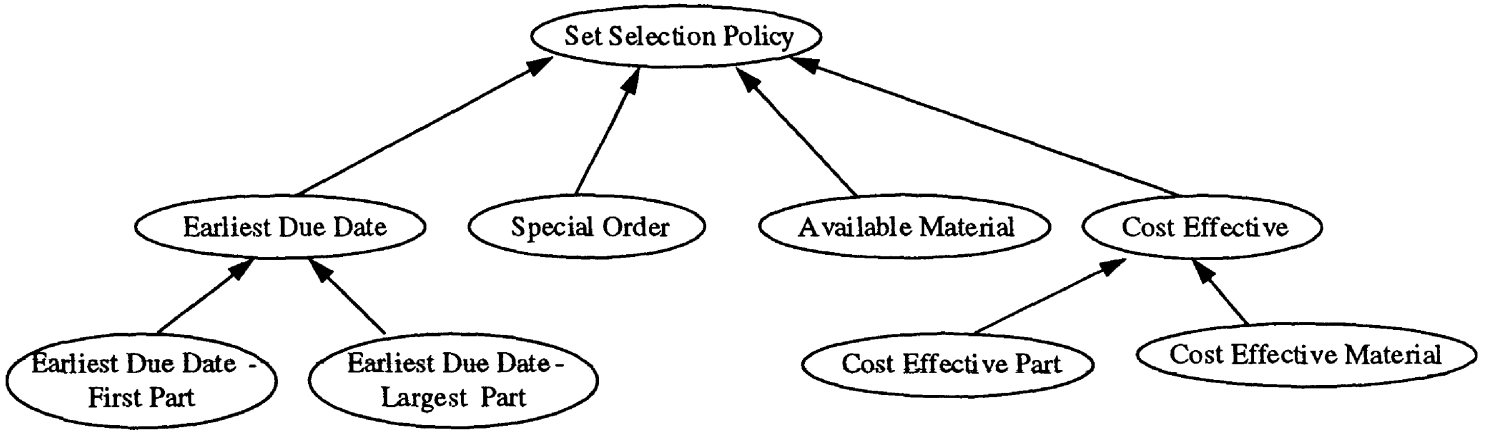

Figure 6 Use Case Extensions of Set Part Selection Policy. 
The dynamic model analyses object interactions. In the Set Selection Policy use case, the actor sets the selection policy. This policy is then reviewed by the Costing actor during cost evaluation, and the policy may be accepted or rejected, or a new policy may be suggested. Similar steps occur in Set Batching and Set Scheduling Policy. The similarity of the event traces and the state diagrams signifies that these three use cases can be derived from one abstract Set or Select Policy use case. The evaluate costs use case, however, is derived from an abstract Evaluation use case in that the costing actor reviews the policies and provides feedback. The two abstract use cases are shown in Figure 7 by ellipses with double lines. The actual use cases specialize (single headed arrow with double lines) the abstract cases, inheriting all of the features. The actor that sets policy and the actor performing evaluation both use Select and the Evaluate.

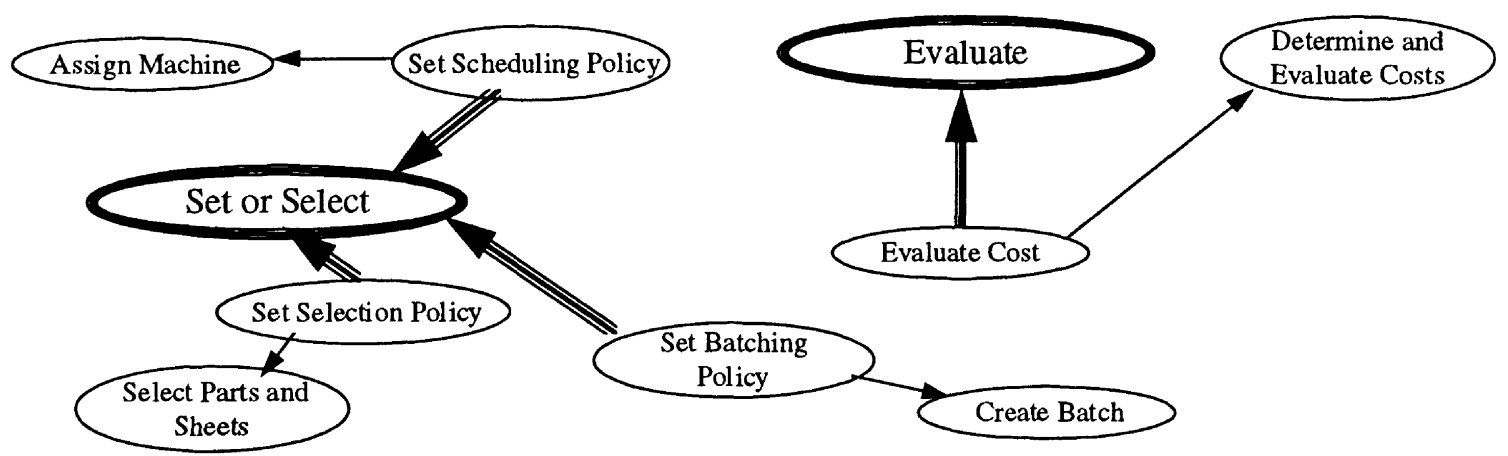

Figure 7 Abstract Use Cases with Inheritance.

\subsection{The Agent Oriented System}

The agent oriented system for the case study consists of four agents that are needed to carry out automated reasoning to set policies and formulate decisions: one each for setting selection, batching, scheduling, and costing policies. Four of the actors from Figure 5 are manifested as the four agents.

Once the agents have been identified, their goals and plans must be established. The major goal of the Selection agent is to set selection policy. The agent's plans arise from the IDEF level 3 diagram in Figure 4 and the use case extensions in Figure 6. Each of these maps directly to a plan. The Selection agent therefore has six plans: earliest due date - first or largest part, special order, part material selection, cost effective part and cost effective sheet.

The Selection agent does not work alone; it collaborates with the Costing agent. This is evident from the fact that both the Selection and the Costing actors use the Set Selection Policy use case, and from the control output from Costing that enters the IDEF level 3 diagram. A plan with collaboration is not intrinsically different from a plan without it. All plans have a set of tasks that are meant to achieve the goal; plans with collaboration follow a script for these tasks. This script can be derived from the event trace for the use case; further, the script is a specialization of the script for the abstract use case.

(Dunskus et al., 1995) discusses the conflicts that can arise between a selector single function agent and an evaluator. If an evaluator gives a negative result, it is a critic. Alternatively a positive evaluator is a praiser, and an evaluator that can make a suggestion is a suggestor. Rather than make these distinctions here, we show that these possibilities are extensions or 
specializations of one coordination protocol. This approach follows (Kuwabara et al., 1995). There are then three possibilities: an evaluator may not have enough information to make an assessment, it may make a positive evaluation, or it may make a negative evaluation or a rejection. If a negative evaluation is made, the evaluator may be able to make a suggestion, adding a fourth possibility.

State diagrams for the coordination protocols or scripts followed by an evaluator agent are shown in Figure 8. In Figure 8a, the evaluator is only able to request information. In Figure $8 \mathrm{~b}$, the evaluator can also be a praiser in that it can accept a selector's findings, or it can be a rejector or a critic. If the evaluator can also be a suggestor, a further orthogonal addition would be made to the state diagram. This script maps directly to the role of the evaluator in the event trace for the use case Evaluate. The corresponding script for the selector agent has states provideInformation, reselect, and rejectGoal.
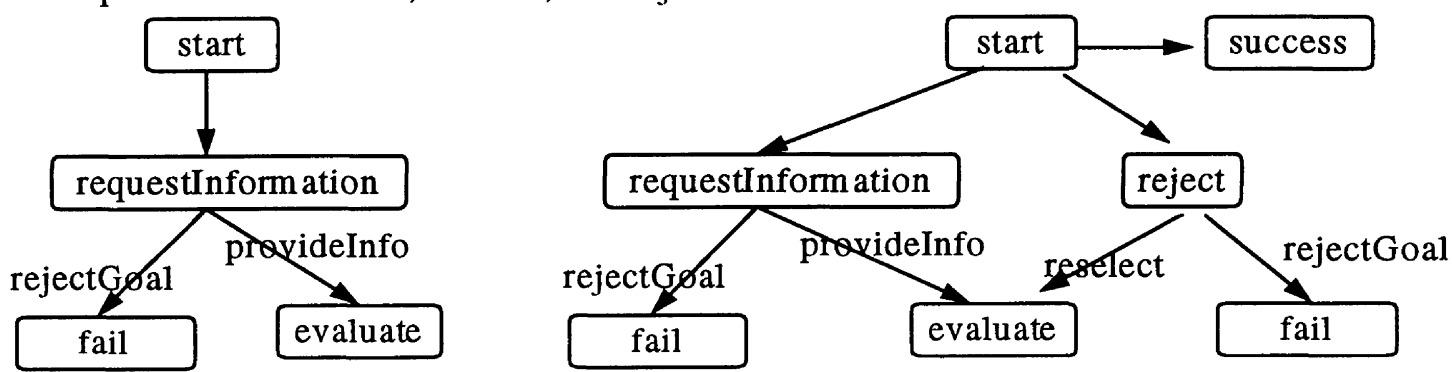

Figure 8a Evaluator Script Figure 8b Evaluator Script with Rejection and Acceptance.

The plans, whether they involve cooperation or not, are enacted or intended based upon strategic knowledge and on context or invocation conditions. The context conditions can be derived from the use case extension conditions and the conditions in the determine policy function of Figure 4. A use case extension is indicated by a probe in the sequence diagram for the original or base use case. When the case reaches the probe, it checks whether the extension condition is true. If it is, the case follows the extension to its completion.

The Selection agent's behavior is enacted by stating a new goal to determine selection policy. This goal is stated if the context has changed, corresponding to a new input (a part, or a material) to the set selection policy function. Once this new goal is posted, the agent decides what plan should be intended based on the conditions that match the probes.

Information must either be retained within the agent as beliefs (passive objects) or obtained from coexisting objects via sensors. Additionally, achieved goals bring about changes to beliefs or modifications manifested via effectors. Parts and material are the objects that concern the selection agent. The agent's sensors monitor part and material features, raising alarms when part or material data meet certain criteria. The agent's effectors impact instances of parts and sheets, resetting or newly defining their priorities.

Sensors and effectors for setting selection policy are sensors to monitor part and material features and watch for special orders. Based on its own knowledge and the control output from Costing, the Selection agent may reject a costly batch or sheet, placing the parts onto the part list and the sheet in inventory. These actions are carried out by effectors. The part selection filter is responsible for prioritizing the parts, according to the agent's intentions, and it is another effector. The sheet selection filter acts similarly. 


\subsection{SUMMARY AND FUTURE WORK}

This paper has described steps toward a methodology for developing agent based systems for enterprise integration. The methodology is based upon the IDEF and CIMOSA modelling frameworks for enterprise integration and on object oriented techniques. With this methodology, agents can be identified, along with their plans, goals, and beliefs, and sensors and effectors. Agent prescriptive collaboration has also been addressed, and the methodology for the analysis and design of the collaboration is based on use cases and use case abstraction.

The agent oriented system for the case study is under development in dMARS and C++. Whereas the intial system utilizes passive objects as beliefs, an integrated agent and object design and implementation is underway. When this work is finished, a more complete statement of the agent development methodology will be formulated.

In addition to the implementation, several areas have been identified for further work: i) Additional forms of collaboration and conflict - agent collaboration is often much more comprehensive than that discussed in this paper; each agent can collaborate with every other agent. Additionally, agent collaboration is not only prescriptive; proscriptive approaches need to be investigated. ii) Opportunistic behavior - pro- active behavior and context conditions do provide for opportunistic behavior, but additional capability is needed. iii) Synchronization behavior - the object models must address concurrency and synchronization. iv) Object oriented designs - designs for sensor, effector, encapsulator, and other objects must be developed. v) Structured and unstructured messaging - between agents and objects.

\subsection{ACKNOWLEDGEMENTS}

This project was partly funded by the Cooperative Research Centre for Intelligent Decision Systems under the Australian Government's Cooperative Research Centres Program.

\subsection{REFERENCES}

Barbuceanu, M. and M. S. Fox (1995) "COOL: A Language for Describing Coordination in Multi-Agent Systems," First International Conference on Multi- Agent Systems, 1995. Booch, G. (1994) Object Oriented Analysis and Design with Applications, Second Edition, The Benjamin/ Cummings Publishing Company, 1994.

Bravoco, R. R., and S. B. Yadav (1985a) "Requirement Definition Architecture - An Overview", Computers in Industry, Vol. 6.

Bravoco, R. R., and S. B. Yadav (1985b) "A Methodology to Model the Functional Structure of an Organization", Computers in Industry, Vol. 6.

Brenton, A. (1995) “The dMARS Plan Language Reference Manual”, Australian Artificial Intelligence Institute (AAII).

Cohen, D., and G. Ringwood (1995) "Decision Table Languages and Guarded Definite Cleauses: Old Wine in New Bottles", Queen Mary and Westfield College, Dept of Computer Science Report.

Cutkosky, M., R. Engelmore, et al. (1992) "PACT: An Experiment in Integrating Concurrent Engineering Systems", IEEE Computer.

Dunskus, B. V., D. L. Grecu, D. C. Brown, and I. Berker (1995) "Using Single Functions Agents to Investigate Conflict", AI in Engineering Design and Manufacturing, special issue in Conflict Management in Multi-Agent Systems. 
Finin, T., J. Weber, et al., (1993)"Specification of the KQML Agent- Communcation Language", The DARPA Knowledge Sharing Initiative External Interfaces Working Group, February.

Genesereth, M., R. E. Fikes, et al (1992) "Knowledge Interchange Format, Version 3.0, Reference Manual," Report Logic- 92- 1, Computer Science Department, Stanford University. Georgeff, M. P., F. Ingrand, et al (1990) "Research on Procedural Reasoning Systems", SRI International.

Gruber, T. R., J. M. Tenenbaum, and J. C. Weber (1992) "Toward a Knowledge Medium for Collaborative Product Development”, in Artificial Intelligence and Design '92, ed. by J. S. Gero, Proceedings of the Second International Conference on Artificial Intelligence in Design. Huntbach, M. M., N. R. Jennings, and G. A. Ringwood (1995)"How Agents Do It In Stream Logic Programming", Proc. First International Conference on Multi- Agent Systems.

Jacobsen, I. (1992) Object- Oriented Software Engineering: A Use Case Driven Approach, Addison Wesley, 1992.

Klein, M. (1991) "Supporting Conflict Resolution in Cooperative Design Systems", in IEEE Transactions on Systems, Man, and cybernetics, Vol. 21, No. 6, November/ December.

Klein, M.(1995) "Business Process (Re-)Engineering: Methodologies and Multi- Agent Technologies," Tutorial at First International Conference on Multi-agent Systems, 1995.

Kuwabara, K., T. Ishida, and N. Osata (1993)“AgenTalk: Describing Multiagent Coordination Protocols with Inheritance," submitted to Tools for Artificial Intelligence Conference, 1995.

Kwok, A., and D. Norrie, (1993) "Intelligent Agent Systems for Manufacturing Applications", J. of Intelligent Manufacturing, Vol 4.

Matsuoka, S., and A. Yonezawa (1993) "Analysis on Inheritance Anomaly in Object-Oriented Concurrent Programming Languages", in Research Directions' in Concurrent Object-Oriented Programming (ed. Gull Agha, P. Wegner, and A. Yonezawa), MIT Press, Cambridge, MA. Pan, J. Y. C., and J. M. Tenenbaum (1991) "An Intelligent Agent Framework for Enterprise Integration”, IEEE Trans.on Systems, Man and Cybernetics, Vol. 21, No. 6, November/ December.

Rumbaugh, J., M. Blaha, et al.(1991) Object Oriented Modeling and Design, Prentice Hall. Tenenbaum, J. M., J. C. Weber, and T. R. Gruber (1992)“'Enterprise Integration: Lessons from SHADE and PACT", in Enterprise Integration Modeling, Proceedings of the First International Conference, Etd. by C. J. Petrie, MIT Press.

Wooldridge, M. J., and N. R. Jennings (1995) “Agent Theories, Architectures, and Languages”, Tutorial, First International Conference on Multi- Agent Systems.

Dr. Elizabeth A. Kendall is a Senior Lecturer in the Department of Computer Systems Engineering, RMIT. Her research activities center on agent based systems and advanced applications of object oriented techniques. She has a B.S. from the Massachusetts Institute of Technology, and a M.S. and Ph.D. from the California Institute of Technology.

Margaret T. Malkoun received the B.S. degree in Computer Science in 1991 from RMIT and is currently undertaking a Ph.D. in Computer Systems Engineering at RMIT. Her research is focused on integration techniques for manufacturing systems and applications of agent-based systems in manufacturing.

Dr. C. Harvey Jiang is a Research Engineer in Computer Systems and Manufacturing Systems Engineering at RMIT. He has a PhD in Manufacturing Systems from RMIT. 